\title{
The novel CD19-targeting antibody-drug conjugate huB4-DGN462 shows improved anti-tumor activity compared to SAR3419 in CD19-positive lymphoma and leukemia models
}

\author{
Stuart W. Hicks, ${ }^{1 *}$ Chiara Tarantelli, ${ }^{2 *}$ Alan Wilhem,${ }^{1}$ Eugenio Gaudio, ${ }^{2}$ \\ Min Li, ${ }^{1}$ Alberto J. Arribas, ${ }^{2}$ Filippo Spriano, ${ }^{2}$ Roberta Bordone, ${ }^{3}$ \\ Luciano Cascione, ${ }^{2,4}$ Katharine C. Lai, ${ }^{1}$ Qifeng Qiu, ${ }^{1}$ Monica Taborelli, ${ }^{5}$ \\ Davide Rossi, ${ }^{2,3}$ Georg Stussi, ${ }^{3}$ Emanuele Zucca, ${ }^{3}$ Anastasios Stathis, ${ }^{3}$ \\ Callum M. Sloss ${ }^{1}$ and Francesco Bertoni ${ }^{2}$
}

\begin{abstract}
*These authors contributed equally to this work.
${ }^{1}$ ImmunoGen Inc., Waltham, MA, USA; ${ }^{2}$ Università della Svizzera italiana, Institute of Oncology Research, Bellinzona, Switzerland; ${ }^{3}$ Oncology Institute of Southern Switzerland, Bellinzona, Switzerland; ${ }^{4}$ Swiss Institute of Bioinformatics, Lausanne, Switzerland and ${ }^{5}$ Cytogenetics Laboratory, Ente Ospedaliero Cantonale, Bellinzona, Switzerland
\end{abstract}

\section{ABSTRACT}

\begin{abstract}
A ntibody-drug conjugates (ADC) are a novel way to deliver potent cytotoxic compounds to cells expressing a specific antigen. Four ADC targeting CD19, including SAR3419 (coltuximab ravtansine), have entered clinical development. Here, we present huB4-DGN462, a novel ADC based on the SAR3419 anti-CD19 antibody linked via sulfoSPDB to the potent DNA-alkylating agent DGN462. huB4-DGN462 had improved in vitro anti-proliferative and cytotoxic activity compared to SAR3419 across multiple B-cell lymphoma and human acute lymphoblastic leukemia cell lines. In vivo experiments using lymphoma xenografts models confirmed the in vitro data. The response of B-cell lymphoma lines to huB4DGN462 was not correlated with CD19 expression, the presence of BCL2 or MYC translocations, TP53 inactivation or lymphoma histology. In conclusion, huB4-DGN462 is an attractive candidate for clinical investigation in patients with B-cell malignancies.
\end{abstract}

\section{Introduction}

Lymphomas are among the most common cancers and causes of cancer-related cell deaths in both adults and children. ${ }^{1}$ In western countries, B-cell lymphomas, and diffuse large B-cell lymphoma (DLBCL) in particular, represent the vast majority of cases. Despite treatment improvements, a proportion of B-cell lymphoma patients still succumb because of chemorefractory disease, requiring the need to find novel therapeutic modalities to extend patients' lives. ${ }^{2,3}$ These neoplastic B cells express many proteins on their cell surface that are potential therapeutic targets. ${ }^{4}$ The clinical success of the anti-CD20 monoclonal antibody, rituximab, demonstrates the potential of targeting B-cell specific surface proteins. ${ }^{5}$ Antibody-drug conjugates $(\mathrm{ADC})$ are an innovative way to deliver potent cytotoxic compounds to cells expressing a specific antigen, as shown by brentuximab vedotin in patients with CD30-positive lymphomas and for ado-trastuzumab emtansine in patients with HER2-positive breast cancer. ${ }^{4}$ Another plasma membrane target, CD19, is almost exclusively expressed on $\mathrm{B}$ cells, appearing at the pre-B-cell stage and remaining expressed until their terminal differentiation into plasma cells ${ }^{6}$ and unlike CD20, CD19 is rapidly internalized, which makes it better suited for ADC development. ${ }^{6-}$ ${ }^{8}$ Four ADC targeting CD19, SAR3419 (coltuximab ravtansine, huB4-DM4), ${ }^{9 \cdot 13}$ SGNCD19A (denintuzumab mafodotin), ${ }^{14,15}$ ADCT-402 (loncastuximab tesirine), ${ }^{16-18}$ and SGN-CD19B ${ }^{19}$ have entered clinical development. SAR3419 is composed of the maytansinoid payload DM4 attached to the anti-CD19 humanized monoclonal
Haematologica 2019

Volume 104(8):1633-1639

\section{Correspondence:}

FRANCESCO BERTONI/CALLUM SLOSS

frbertoni@mac.com/

callum.sloss@immunogen.com

Received: November 3, 2018.

Accepted: February 7, 2019.

Pre-published: February 7, 2019.

doi:10.3324/haematol.2018.211011

Check the online version for the most updated information on this article, online supplements, and information on authorship \& disclosures: www.haematologica.org/content/104/8/1633

(c)2019 Ferrata Storti Foundation

Material published in Haematologica is covered by copyright. All rights are reserved to the Ferrata Storti Foundation. Use of published material is allowed under the following terms and conditions:

https://creativecommons.org/licenses/by-nc/4.0/legalcode. Copies of published material are allowed for personal or internal use. Sharing published material for non-commercial purposes is subject to the following conditions:

https://creativecommons. org/licenses/by-nc/4.0/legalcode, sect. 3. Reproducing and sharing published material for commercial purposes is not allowed without permission in writing from the publisher. 
IgG1 antibody, huB4, via an N-succinimidyl-4-(2pyridyldithio)butyrate (SPDB) cleavable linker. ${ }^{9}$ SAR3419 demonstrated clinical activity in patients with relapsed/refractory B-cell lymphoma as both a single agent and in combination with rituximab. ${ }^{10-13}$ To further, improve the clinical benefit of targeting CD19, second generation $\mathrm{ADC}$ can be developed incorporating new innovative linkers and payload moieties. ${ }^{20}$ For example, the addition of a sulfonate group in the disulfide linker SPDB ([N-succinimidyl 2-sulfo-4-(2-pyridyldithio)butanoate], sulfo-SPDB) can increase the potency of ADC bearing similar payloads. ${ }^{21}$ The indolinobenzodiazepine pseudodimer DGN462 is a potent DNA-alkylating agent with proven anti-tumor activity in preclinical models of solid tumors and acute myeloid leukemia (AML). ${ }^{22}$ These two innovations have been implemented in the recently developed IMGN779, an anti-CD33 ADC with promising preclini$\mathrm{cal}^{23,24}$ and early clinical activity ${ }^{25}$ in AML. Here, we present huB4-DGN462, a novel ADC utilizing the huB4, antiCD19 antibody, linked via sulfo-SPDB to DGN462. The huB4-ADC incorporating sulfo-SPDB and DGN462 demonstrated improved in vitro and in vivo activity in lymphoma and leukemia models.

\section{Methods}

\section{Cell lines}

Lymphoma cell lines, all validated for their identity by short tandem repeat DNA fingerprinting (IDEXX BioResearch, Ludwigsburg, Germany), were used and cultured as previously described. ${ }^{26} B C L 2, M Y C$ and TP53 status were defined as reported in Online Supplementary Table S1. Additional cell lines used included the Burkitt's lymphoma Ramos (ACC-603; DSMZ), and the human acute lymphoblastic leukemia (ALL) cell lines RS4;11 (CRL-1873, ATCC), TOM-1 (ACC-578, DSMZ), and BALL-1 (JCRB0071, JCRB).

\section{Antibody-drug conjugates and free payload}

Antibody-drug conjugates (SAR3419, huB4-DGN462, non-targeting IgG-DGN462 control) and cell-permeable unconjugated toxin S-Methyl-DGN462 (DGN462-SMe) were generated by ImmunoGen. huB4-DGN462 and IgG-DGN462 were produced as described previously. ${ }^{22}$ Briefly, a 10-fold molar excess of in situ mixture containing DGN462 and sulfo-SPDB was added to huB4 or IgG antibody in buffer (50 mM EPPS [4-(2-Hydroxyethyl)-1piperazinepropanesulfonic acid], $\mathrm{pH}$ 8.5) containing $15 \%$ dimethylacetamide. Upon completion of conjugation, the reaction mixtures were purified and buffer exchanged into $20 \mathrm{mM}$ histidine, $50 \mathrm{mM}$ sodium chloride, $8.5 \% \mathrm{w} / \mathrm{v}$ sucrose, $0.01 \%$ Tween-20, $50 \mu \mathrm{M}$ sodium bisulfite $\mathrm{pH} 6.2$ using NAP desalting columns (Illustra Sephadex G-25, GE Healthcare).

\section{In vitro anti-tumor activity}

To determine in vitro cytotoxicity potency, B-cell lymphoma and B-ALL cell lines were treated with a 3 -fold dilution series of conjugate for five days without or with a 100 -fold concentration of unconjugated huB4 blocking antibody. The relative number of viable cells in each well was then determined using the WST-8 based Cell Counting Kit-8 (Dojindo Molecular Technologies Inc., Rockville, MD, USA). The surviving fraction of cells was plotted against conjugate concentration and the EC50 of activity was calculated using a non-linear regression analysis (GraphPad Prims 4.0). For higher-throughput in vitro cytotoxicity screening, lymphoma or leukemia cells $\left(10^{4}\right)$ were seeded in 96-well plates and the indicated compounds were added to create 4-fold dilution series ranging from $200 \mathrm{nM}$ to $0.19 \mathrm{pM}$ and assayed by MTT following 72 hours (h) of treatment, as previously described. ${ }^{24}$

Apoptosis was assessed using the luminescence-base Caspase-Glo 3/7 assay kit (Promega) according to the manufacturer's instructions. Briefly, cells were seeded in 384-well plates, treated with $\mathrm{ADC}$ or free toxin at the following concentrations (50 and 1,000 pM for huB4-DGN462 and DGN462-SMe; 100 and 5,000 pM for SAR3419). Apoptosis was defined by at least a 1.5-fold increase in signal activation with respect to controls. Differences among groups were calculated using the Wilcoxon rank-sum test (Stata/SE 12.1 for Mac, Stata Corporation, College Station, TX, USA). $P<0.05$ was considered statistically significant.

\section{CD19 expression}

Fluorescence-activated cell sorting (FACS) for CD19 antigen expression analysis was performed on fresh low passage lymphoma cell lines. Cells were washed with ice-cold FACS buffer $(\mathrm{PBS}+0.5 \% \mathrm{BSA})$ and divided $1 \times 10^{6}$ cells/tube. A pretreatment with human FcR blocking (Miltenyi Biotec Inc., Auburn, CA, USA) was performed according to the manufacturer's instructions. CD19-PE $(5 \mu \mathrm{L} ; 1 \mu \mathrm{g})$ or control isotype was incubated with cells at $4^{\circ} \mathrm{C}$ for 30 minutes. Cells were washed twice and re-suspended in FACS buffer. Flow-cytometry analysis was carried out with a FACS Canto II instrument (BD Biosciences). Median Fluorescence intensity (MFI) of each sample was determined using FacsDiva v.8.0.1 software (BD Biosciences, Allschwil, Switzerland). Unstained cells and cells stained with isotype control antibody were used as controls. RNA expression levels, obtained with the HumanHT-12 v.4 Expression BeadChip (Illumina, San Diego, CA, USA) and with the HTG EdgeSeq Oncology Biomarker panel (HTG Molecular Diagnostics Inc., Tucson, AZ, USA) were published previously. ${ }^{26}$ For leukemia cell lines, the median number of CD19 antibody binding sites (ABC) was determined as previously described. ${ }^{24}$ Pairwise correlations were assessed using Stata/SE 12.1 for Mac. $P<0.05$ was considered statistically significant.

\section{In vivo experiments}

Experiments using subcutaneous (DOHH2) and disseminated (Farage) xenograft models in female CB.17 severe combined immunodeficient (SCID) mice (Charles River Laboratories, Wilmington, MA, USA) were run following previously described procedures. ${ }^{27}$ All animal procedures were performed in strict accordance with Immunogen's Animal Care and Use Committee and the National Institutes of Health Guide for the Care and Use of Laboratory Animals. For the DOHH2 model, mice were inoculated subcutaneously with $10^{7}$ cells and were randomized by tumor volume (TV) into treatment groups (6 mice/group), when the average TV reached $100 \mathrm{~mm}^{3}$. Tumor volumes were recorded 2-3 times weekly by caliper measurements of the height $(\mathrm{H})$, length $(\mathrm{L})$, and width $(\mathrm{W})$ of the tumor, following the formula:

$$
\mathrm{TV}=(\mathrm{H} \times \mathrm{L} \times \mathrm{W}) / 2
$$

For the disseminated model, mice were inoculated intravenously (IV) via the lateral tail vein with $10^{7}$ Farage cells and were randomized into treatment groups ( 8 mice/group) by body weight. Animal survival was followed; animals were removed from the study when advanced health distress signs were observed or measured (> $20 \%$ loss of body weight, hind leg paralysis, tumor growth on body, or moribund state). 


\section{Results}

\section{huB4-DGN462 is a novel anti-CD19 antibody-drug conjugate}

The humanized anti-CD19 antibody huB4 ${ }^{28}$ was conjugated with DGN462, a novel member of the DNA-alkylating indolinobenzodiazepine pseudodimers (IGNs) class $^{22}$ via the cleavable disulfide linker, sulfo-SPDB, at a drug-toantibody ratio (DAR) of 2.8 (Figure 1A). This novel CD19targeting ADC, huB4-sulfo-SPDB-DGN462 or huB4-
DGN462, combines the anti-CD19 antibody used in SAR3419 (Figure 1B) ${ }^{9}$ and the potent linker/payload exploited in the anti-CD33 IMGN779. ${ }^{24}$ To evaluate the consequence of conjugation on antigen binding, the relative binding affinity of huB4-DGN462 and the unconjugated huB4 antibody to CD19 was determined by FACS analysis on the Burkitt's lymphoma Ramos cell line, which endogenously express human CD19 (Figure 1C). The huB4-DGN462 bound with similar high affinity (KD $\sim 0.15 \mathrm{nM}$ ) as the unconjugated antibody, suggesting that

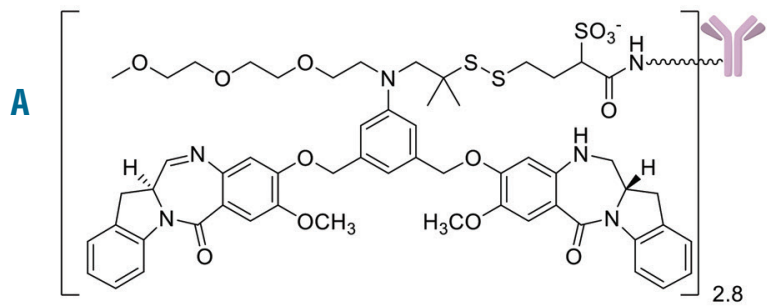

huB4-DGN462
(huB4-sulfo-SPDB-DGN462)

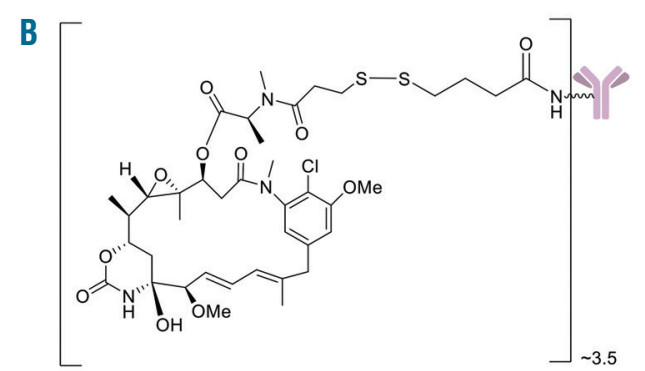

SAR3419

(huB4- SPDB-DM4)

\section{C}
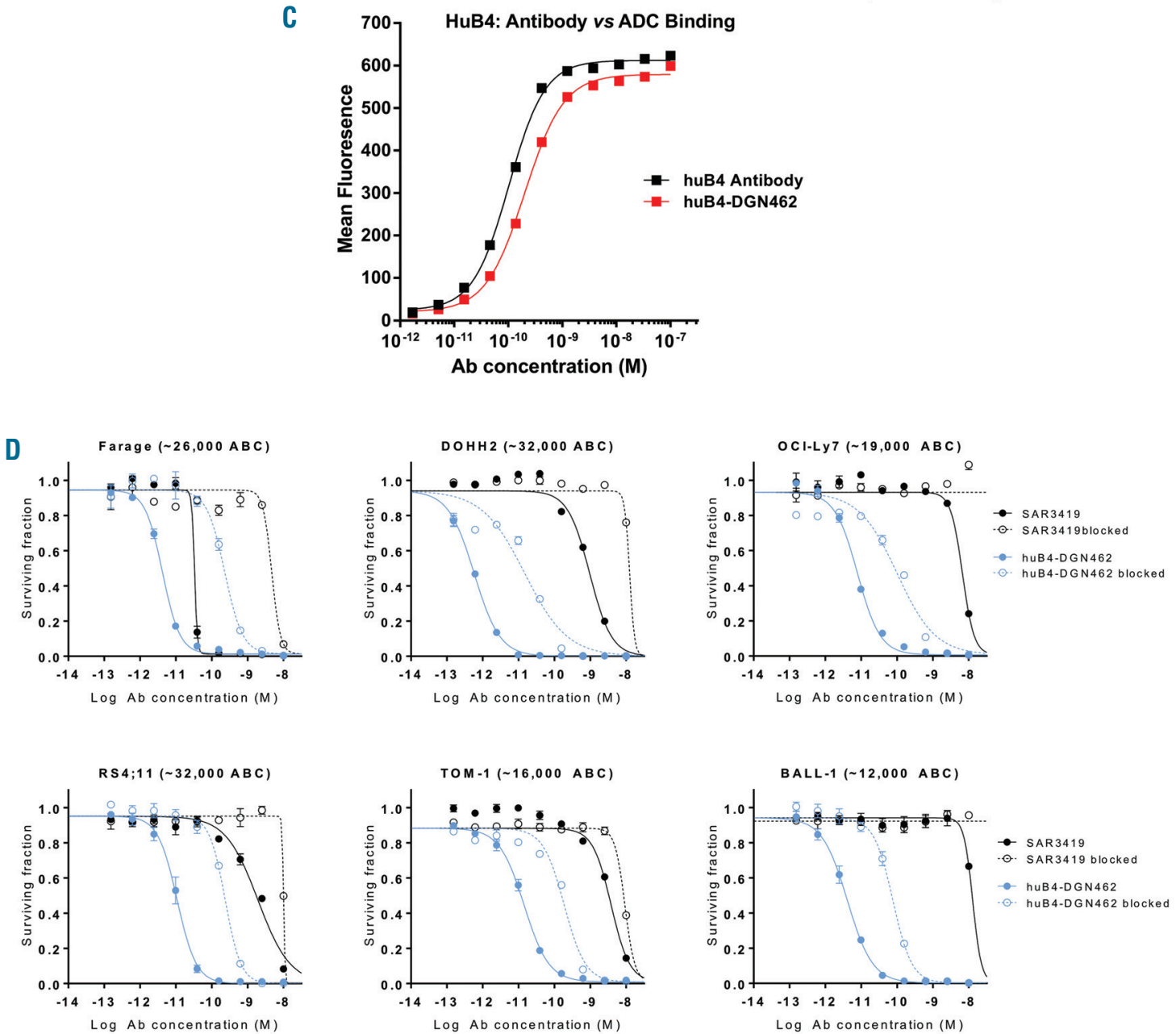

Figure 1. huB4-DGN462 is a novel anti-CD19 antibody-drug conjugate (ADC). (A) Chemical structure of huB4-DGN462. (B) Chemical structure of SAR3419. (C) Relative binding affinities of huB4 and huB4-DGN462 on Ramous cells. Cells were cultured with the indicated concentrations of either the unconjugated antibody or intact ADC, and binding was detected by flow cytometry using a fluorescently labeled anti-human antibody. (D) In vitro potency of huB4-DGN462 and SAR3419 in diffuse large B-cell lymphoma (DLBCL) (top) or B-cell acute lymphoblastic leukemia (B-ALL) (bottom) cell lines with or without blocking antibody. ABC: antibodies bound per cell. 
Table 1. Anti-tumor activity of huB4-DGN462 and SAR3419 in B-cell lymphoma cell lines.

\begin{tabular}{|c|c|c|c|c|c|c|}
\hline & \multirow[b]{2}{*}{ Number of cell lines } & \multicolumn{2}{|c|}{ huB4-DGN462 } & \multicolumn{2}{|c|}{ SAR3419 } & \multirow[b]{2}{*}{ Whlcoxon rank-sum $P$} \\
\hline & & Median IC50 & $95 \%$ Cl & Median IC50 & $95 \%$ CI & \\
\hline All & 46 & $100 \mathrm{pM}$ & $38-214 \mathrm{pM}$ & $37 \mathrm{nM}$ & $2.4-5.1 \mathrm{nM}$ & $<0.0001$ \\
\hline GCB-DLBCL & 19 & $100 \mathrm{pM}$ & 3 pM-1170 pM & $2 \mathrm{nM}$ & $0.7-3.6 \mathrm{nM}$ & 0.003 \\
\hline ABC-DLBCL & 8 & $600 \mathrm{pM}$ & 20 pM-3000 pM & $6.5 \mathrm{nM}$ & $3.8-7.3 \mathrm{nM}$ & 0.001 \\
\hline MCL & 10 & $45 \mathrm{pM}$ & $5-84 \mathrm{pM}$ & $3 \mathrm{nM}$ & $1.3-9.7 \mathrm{nM}$ & 0.017 \\
\hline MZL & 6 & $65 \mathrm{pM}$ & $6-910 \mathrm{pM}$ & $5 \mathrm{nM}$ & $1.5-9.8 \mathrm{nM}$ & 0.004 \\
\hline CLL & 2 & $2250 \mathrm{pM}$ & $1500-3000 \mathrm{pM}$ & $25 \mathrm{nM}$ & $10-40 \mathrm{nM}$ & 0.130 \\
\hline PMBCL & 1 & $200 \mathrm{pM}$ & n.d. & $10 \mathrm{nM}$ & n.d. & n.d. \\
\hline
\end{tabular}

GCB-DLBCL: germinal center B-cell type diffuse large B-cell lymphoma; ABC-DLBCL: activated B-cell like diffuse large B-cell lymphoma; MCL: mantle cell lymphoma; MZL: marginal zone lymphoma; CLL: chronic lymphocytic leukemia; PMBCL: primary mediastinal large B-cell lymphoma; n.d.: not determined.

the incorporation of the payload did not appreciably alter antibody binding affinity.

\section{huB4-DGN462 has improved in vitro anti-lymphoma activity compared to SAR3419}

To characterize the ability of huB4-DGN462 to kill tumor cells, the in vitro cytotoxicity of huB4-DGN462 was compared to SAR3419 in a subset of B-cell lymphoma and B-ALL tumor cell lines. The tumor cell killing was assayed five days after treatment. While SAR3419 had limited potency, huB4-DGN462 was highly active with IC50s ranging from 1-16 pM (Figure 1D). In addition, unconjugated antibody was able to block the activity of both huB4-DGN462 and SAR3419 confirming that the cytotoxicity of both conjugates is the result of specific CD19 antigen binding on tumor cells.

To further characterize the in vitro potency of huB4DGN462, we performed a 72-h in vitro cytotoxicity screen on 46 B-cell lymphoma cell lines, mostly derived from DLBCL, exposed also to SAR3419 (Table 1 and Online Supplementary Table S1). huB4-DGN462 was potent with a median IC50 of $100 \mathrm{pM}$ (95\%CI: 38-214). The free payload, DGN462-SMe, had a median IC50 of $26 \mathrm{pM}$ (95\%CI: 1-186) (Online Supplementary Table S1). huB4DGN462 induced caspase 3/7 activation consistent with an apoptotic mechanism of action (Online Supplementary Table S1). In agreement, cell cycle analysis in two DLBCL cell lines showed a G2-M arrest with subG0 accumulation of cells after huB4-DGN462 and DGN462-SMe treatment (Online Supplementary Figure S1), similar to that reported for IMGN779, a DGN462-containing ADC. ${ }^{24}$

While the rank order of anti-proliferative activity for huB4-DGN462 and SAR3419 was similar across the cell lines tested $(\mathrm{R}=0.42, P=0.004)$ (Online Supplementary Figure S2), huB4-DGN462 was over 30-times more potent than SAR3419 $(P<0.0001)$ (Table 1 and Online Supplementary Figure S3). Increased cytotoxic activity of huB4-DGN462 was also confirmed in six CD19+ B-ALL cell lines (Online Supplementary Table S2).

While a moderate correlation was seen for SAR3419, the response of B-cell lymphoma lines to huB4-DGN462 was not correlated with either surface CD19 protein expression (as measured by FACS) or CD19 RNA expression (as measured by microarray or by targeted RNA-Seq) (Online Supplementary Figures S4 and S5). However, the activity of huB4-DGN462 was positively correlated with the potency of its free payload $(\mathrm{R}=0.71, P<0.0001)$.

Among DLBCL cell lines, the sensitivity to the two
ADCs was not affected by TP53 inactivation (present in 16 of 23 cell lines), nor the presence of MYC or BCL2 translocations (detected in 8 of 24 and 13 of 21 cell lines, respectively). The sensitivity to SAR3419, but not the novel $\mathrm{ADC}$, was lower in activated $\mathrm{B}$-cell-like $(\mathrm{ABC})$ than in germinal center B-cell type (GCB) DLBCL cell lines $(P=0.02)$ (Table 1, Online Supplementary Table S1 and Online Supplementary Figure S6A) This was in agreement with the correlation between SAR3419 activity and CD19 expression and an observed higher CD19 expression in GCB than ABC DLBCL cell lines (Online Supplementary Figure $S 6 B)$.

\section{huB4-DGN462 has superior in vivo anti-lymphoma activity compared to SAR3419}

To examine whether the enhanced antitumor activity of huB4-DGN462 observed in vitro would translate to improved efficacy in vivo, the anti-tumor activity of both of CD19-targeting ADC was evaluated in two DLBCL xenograft models. In the subcutaneous DoHH2 model, a single intravenous dose of huB4-DGN462 resulted in a significant, dose-dependent tumor growth delay and survival benefit at $1.7 \mathrm{mg}$ antibody $(\mathrm{Ab}) / \mathrm{kg}$ (5 of 6 partial responses, 3 of 6 complete responses, 3 of 6 tumor-free survivors) compared to a non-targeted control IgG-DGN462 ADC (1 of 6 partial responses, 1 of 6 complete responses, 1 of 6 tumorfree survivors) (Figure $2 \mathrm{~A}$ ). The anti-tumor activity of huB4DGN462 was better than that obtained in a similar experiment using SAR3419 given at higher doses. Both huB4DGN462 and SAR3419 were well-tolerated, with no significant loss of body weight observed (Online Supplementary Figure S7). In the disseminated Farage model, the $1.7 \mathrm{mg} / \mathrm{kg}$ dose of the non-specific IgG1-DGN462 control conjugate was inactive while there was a significant dose-dependent increase in survival observed in mice treated with as low as $0.17 \mathrm{mg} \mathrm{Ab} / \mathrm{kg}$ of huB4-DGN462 (Figure 2B). At $1.7 \mathrm{mg}$ $\mathrm{Ab} / \mathrm{kg}$, survival of tumor bearing mice was increased over $400 \%$. As above, the efficacy of huB4-DGN462 was better than that achieved with SAR3419. Taken together and consistent with the in vitro findings, treatment with huB4DGN462 resulted in a substantial enhancement of antitumor activity compared to SAR3419.

\section{Discussion}

Here we have compared the anti-lymphoma activity of a novel CD19-targeting ADC, huB4-DGN462, with 
SAR3419, an ADC that demonstrated clinical activity as single agent ${ }^{10,29}$ and in combination with the anti-CD20 monoclonal antibody rituximab. ${ }^{13}$ SAR3419 comprises the maytansinoid microtubule disruptor, DM4, conjugated to the huB4 anti-CD19 antibody via the hydrophobic linker SPDB. ${ }^{6,9}$ Utilizing the same anti-CD19 antibody in SAR3419, we created huB4-DGN462, which incorporates the ultra-potent DNA-alkylating payload, DGN462 linked by the more hydrophilic sulfo-SPDB linker. This sulfoSPDB-DGN462 linker/payload combination has previously been shown to have significantly greater potency (up to 3 logs) than the SPDB-DM4. ${ }^{22}$ As predicted, huB4DGN462 was more potent than SAR3419 in in vitro antiproliferative and apoptotic assays as well as in in vivo
A

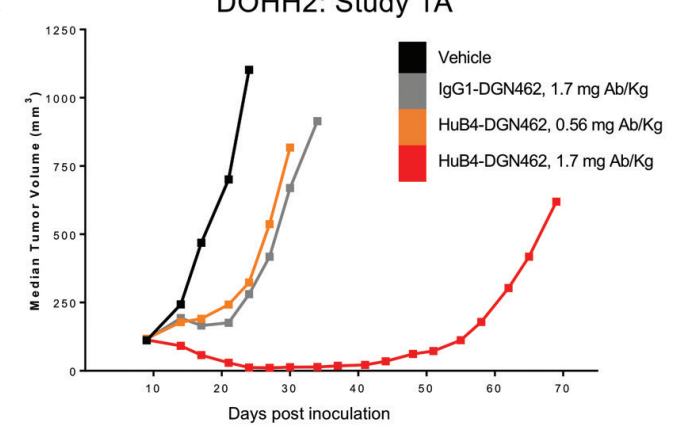

DOHH2: Study 1B

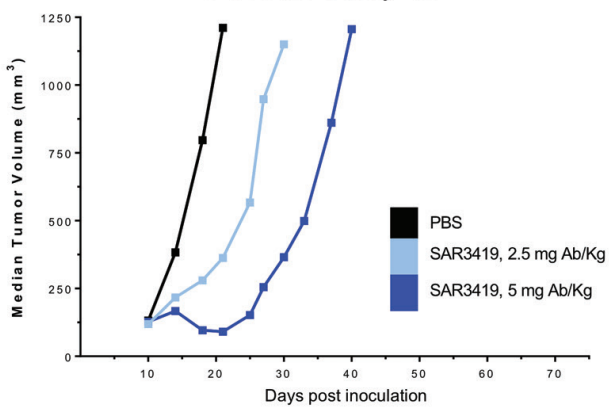

\begin{tabular}{|l|l|c|c|c|c|}
\hline & \multicolumn{1}{|c|}{ Group } & Ab Dose $(\mathbf{m g} / \mathbf{K g})$ & $\% \mathrm{~T} / \mathrm{C}$ & \multicolumn{2}{c|}{ Regressions } \\
\hline & & & & PR & CR \\
\hline Study 1a & Vehicle & - & - & & $1 / 6$ \\
\hline & IgG1-DGN462 & 1.7 & $25 \%$ & $1 / 6$ & $0 / 6$ \\
\hline & HuB4-DGN462 & 0.56 & $29 \%$ & $5 / 6$ & $3 / 6$ \\
\hline & HuB4-DGN462 & 1.7 & $1 \%$ & & $1 / 8$ \\
\hline Study 1b & Vehicle & - & - & $1 / 8$ & $2 / 8$ \\
\hline & SAR3419 & 2.5 & $11 \%$ & $3 / 8$ & \\
\hline & SAR3419 & 5 & $9 \%$ & & \\
\hline
\end{tabular}

B

Farage: Study $1 \mathrm{~A}$

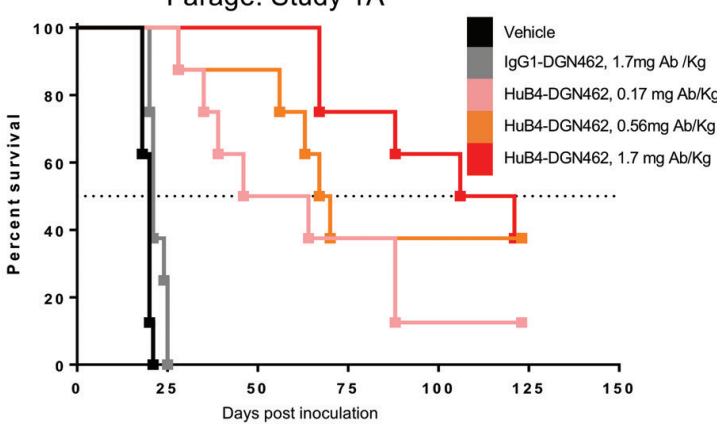

Farage: Study 1B

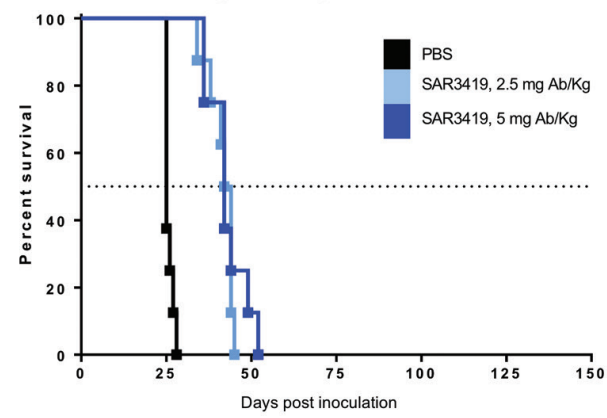

\begin{tabular}{|l|l|c|c|c|c|}
\hline & \multicolumn{1}{|c|}{ Group } & Ab Dose (mg/Kg) & $\begin{array}{c}\text { Median Survival } \\
\text { (Days) }\end{array}$ & $\begin{array}{c}\text { Tumor Growth } \\
\text { Delay/T-C (Days) }\end{array}$ & $\begin{array}{c}\text { \% Increased Life } \\
\text { Span }\end{array}$ \\
\hline Study 1a & Vehicle & - & 20 & - & - \\
\hline & IgG1-DGN462 & 1.7 & 21 & 1 & 5 \\
\hline & HuB4-DGN462 & 0.17 & 55 & 35 & 175 \\
\hline & HuB4-DGN462 & 0.56 & 69 & 49 & 243 \\
\hline & HuB4-DGN462 & 1.7 & 114 & 94 & 468 \\
\hline Study 1b & Vehicle & - & 25 & - & - \\
\hline & SAR3419 & 2.5 & 43 & 18 & 72 \\
\hline & SAR3419 & 5 & 42 & 17 & 68 \\
\hline
\end{tabular}

Figure 2. huB4-DGN462 exhibits more potent in situ antitumor efficacy than SAR3419 in both disseminated (A) and subcutaneous (B) germinal center B-cell type diffuse large B-cell lymphoma (GCB DLBCL) xenograft models. (A) Nude mice bearing Farage xenografts were treated with vehicle, a single dose of huB4-DGN462 (0.17, 0.56 or $1.7 \mathrm{mg}$ antibody / kg), or a single dose of non-targeting lgG1-DGN462 (1.7 mg antibody /kg), as indicated. (Right) Nude mice bearing Farage xenografts were treated with vehicle or a single dose of SAR3419 (2.5 or $5 \mathrm{mg}$ antibody / $\mathrm{kg}$ ) as indicated. The table summarizes the data for the experiments. (B) Nude mice with subcutaneous DOHH2 xenografts were treated with vehicle, a single dose of huB4-DGN462 (0.56 or $1.7 \mathrm{mg}$ antibody $/ \mathrm{kg}$ ), or a single dose of non-targeting IgG1-DGN462 (1.7 mg antibody /kg), as indicated. (Right) Nude mice bearing DOHH2 xenografts were treated with vehicle or a single dose of SAR3419 (2.5 or 5 mg antibody $/ \mathrm{kg}$ ) as indicated. The table summarizes the data for the experiments. Ab: antibody. T/C: treated versus control. PR: partial response (defined when the tumor volume at any given measurement point was $<50 \%$ of the initial pretreatment tumor volume). ${ }^{27} \mathrm{CR}$ : complete response (defined when no palpable tumor could be detected). ${ }^{27}$ TGD: tumor growth delay. 
DLBCL subcutaneous and systemic tumor models. The improved activity of huB4-DGN462 compared to SAR3419 can be attributed to the increased potency of the linker/payload combination of sulfo-SPDB-DGN462 compared to SPDB-DM4.

HuB4-DGN462 was highly active in multiple cell lines derived from B-cell lymphomas. The antitumor activity was not affected by the lymphoma histology or DLBCL subtype or TP53, BCL2 or MYC gene status. Despite specificity for CD19, similar to other ADC, the anti-lymphoma activity of huB4-DGN462 did not correlate with the absolute expression level of CD19, ${ }^{30,31}$ but rather the sensitivity of the cell line to the free payload. The impressive potency of huB4-DGN462 observed is likely to be due to the fact that low target expression was sufficient to deliver a cytotoxic concentration of the novel linker/payload combination. Given the broad expression profile of CD19 in B-cell malignancies, CD19 is an attractive target for cancer therapy. In particular, CD19-targeting immunotherapies have seen recent successes with the US Food and Drug Administration (FDA) approvals of the bispecific T-cell engaging antibody blinatumomab (Blincyto) ${ }^{32-34}$ and the FDA approvals of two chimeric antigen receptors (CAR) T-cell therapies targeting CD19, tisagenlecleucel (Kymriah) ${ }^{35}$ and axicabtagene cilolecucel
(Yescarta). ${ }^{36}$ Despite the success of CD19-targeting immunotherapies, there still remains the need for more effective therapies, particularly in patients with relapsed/refractory lymphomas such as DLBCL. The clinical activity of CD19-targeting ADC following other antiCD19 directed therapeutic modalities ${ }^{32-39}$ will have to be determined. However, unlike immunotherapies that stimulate the patient's immune systems to fight cancer, $\mathrm{ADC}$ like huB4-DGN462 kill tumor cells by delivering a cytotoxic payload directly to tumor cells. Given the distinct difference in mechanism of action of ADC to that of immunotherapies, patients have the potential to benefit from both types of therapies.

This work demonstrates that huB4-DGN462 has superior preclinical antitumor activity compared to clinicallyvalidated SAR3419. Each of the individual components of huB4-DGN462 (antibody, linker and payload) have passed early clinical evaluation ${ }^{13,25}$ making huB4-DGN462 an attractive candidate for clinical investigation with the potential to extend the lives of patients with B-cell malignancies.

\section{Funding}

Partially supported by institutional research funds from ImmunoGen and the Gelu Foundation (to FB).

\section{References}

1. Siegel RL, Miller KD, Jemal A. Cancer statistics, 2018. CA Cancer J Clin. 2018; 68(1):7-30

2. Iacoboni G, Zucca E, Ghielmini M, Stathis A. Methodology of clinical trials evaluating the incorporation of new drugs in the firstline treatment of patients with diffuse large B-cell lymphoma (DLBCL): a critical review. Ann Oncol. 2018;29(5):1120-1129.

3. Stathis A, Iasonos A, Seymour JF, et al. Report of the $14^{\text {th }}$ International Conference on Malignant Lymphoma (ICML) Closed Workshop on Future Design of Clinical Trials in Lymphomas. Clin Cancer Res. 2018;24(13):2993-2998.

4. Moek KL, de Groot DJA, de Vries EGE, Fehrmann RSN. The antibody-drug conjugate target landscape across a broad range of tumour types. Ann Oncol. 2017; 28(12):3083-3091.

5. Karmali R, Kimby E, Ghielmini M, Flinn IW, Gordon LI, Zucca E. Rituximab: a benchmark in the development of chemotherapy-free treatment strategies for follicular lymphomas. Ann Oncol. 2018; 29(2):332-340.

6. Blanc V, Bousseau A, Caron A, Carrez C, Lutz RJ, Lambert JM. SAR3419: an antiCD19-Maytansinoid Immunoconjugate for the treatment of B-cell malignancies. Clin Cancer Res. 2011;17(20):6448-6458.

7. Nathwani N, Chen R. Antibody drug conjugates in lymphoma. Clin Investig (Lond). 2014;6(10):915-922.

8. Gerber HP, Kung-Sutherland M, Stone I, et al. Potent antitumor activity of the antiCD19 auristatin antibody drug conjugate hBU12-vcMMAE against rituximab-sensitive and -resistant lymphomas. Blood. 2009;113(18):4352-4361.

9. Hong EE, Erickson H, Lutz RJ, et al. Design of coltuximab ravtansine, a CD19-targeting antibody-Drug Conjugate (ADC) for the treatment of B-cell malignancies: structureactivity relationships and preclinical evaluation. Mol Pharm. 2015;12(6):1703-1716.

10. Younes A, Kim S, Romaguera J, et al. Phase I multidose-escalation study of the antiCD19 maytansinoid immunoconjugate SAR3419 administered by intravenous infusion every 3 weeks to patients with relapsed/refractory B-cell lymphoma. J Clin Oncol. 2012;30(22):2776-2782.

11. Ribrag V, Dupuis J, Tilly H, et al. A doseescalation study of SAR3419, an anti-CD19 antibody maytansinoid conjugate, administered by intravenous infusion once weekly in patients with relapsed/refractory B-cell non-Hodgkin lymphoma. Clin Cancer Res. 2014;20(1):213-220.

12. Trneny M, Verhoef G, Dyer MJ, et al. Starlyte phase II study of coltuximab ravtansine (CoR, SAR3419) single agent: Clinical activity and safety in patients (pts) with relapsed/refractory $(\mathrm{R} / \mathrm{R})$ diffuse large B-cell lymphoma (DLBCL; NCT01472887). J Clin Oncol. 2014;32(15_suppl):8506-8506.

13. Coiffier B, Thieblemont C, de Guibert S, et al. A phase II, single-arm, multicentre study of coltuximab ravtansine (SAR3419) and rituximab in patients with relapsed or refractory diffuse large B-cell lymphoma. Br J Haematol. 2016;173(5):722-730.

14. Law C-L, Sutherland M, Miyamoto J, et al. Abstract 625: Preclinical characterization of an auristatin-based anti-CD19 drug conjugate, SGN-19A. Cancer Res. 2011;71(8 Supplement):625-625.

15. Chen RW, Jacobsen ED, Kostic A, Liu T, Moskowitz CH. A randomized, phase 2 trial of denintuzumab mafodotin and RICE vs RICE alone in the treatment of patients (pts) with relapsed/refractory $(r / r)$ diffuse large B-cell lymphoma (DLBCL) who are candidates for autologous stem cell transplant (ASCT). J Clin Oncol. 2016; 34(15_suppl):TPS7584-TPS7584.

16. Zammarchi F, Corbett S, Adams L, et al.
ADCT-402, a PBD dimer-containing antibody drug conjugate targeting CD19expressing malignancies. Blood. 2018; 131(10):1094-1105.

17. Caimi P, Kahl BS, Hamadani M, et al. Safety and efficacy of Adct-402 (loncastuximab tesirine), a novel antibody drug conjugate, in relapsed/refractory follicular lymphoma and mantle cell lymphoma: interim results from the Phase 1 first-in-human Study. Blood. 2018;132(Suppl 1):2874-2874.

18. Radford J, Kahl BS, Hamadani M, et al Interim Results from the First-in-Human Clinical Trial of Adct-402 (Loncastuximab Tesirine), a Novel PyrrolobenzodiazepineBased Antibody Drug Conjugate, in Relapsed/Refractory Diffuse Large B-Cell Lymphoma. Blood. 2018;132(Suppl 1):398398.

19. Ryan MC, Palanca-Wessels MC, Schimpf $\mathrm{B}$, et al. Therapeutic potential of SGNCD19B, a PBD-based anti-CD19 drug conjugate, for treatment of $\mathrm{B}$-cell malignancies. Blood. 2017;130(18):2018-2026.

20. Beck A, Goetsch L, Dumontet C, Corvaia $\mathrm{N}$. Strategies and challenges for the next generation of antibody-drug conjugates. Nat Rev Drug Discov. 2017;16(5):315-337.

21. Zhao RY, Wilhelm SD, Audette C, et al Synthesis and evaluation of hydrophilic linkers for antibody-maytansinoid conjugates. J Med Chem. 2011;54(10):3606-3623.

22. Miller ML, Fishkin NE, Li W, et al. A New Class of Antibody-Drug Conjugates with Potent DNA Alkylating Activity. Mol Cancer Ther. 2016;15(8):1870-1878.

23. Whiteman KR, Noordhuis P, Walker R, et al. The Antibody-Drug Conjugate (ADC) IMGN779 Is Highly Active in Vitro and in Vivo Against Acute Myeloid Leukemia (AML) with FLT3-ITD Mutations. Blood. 2014:124(21):2321-2321.

24. Kovtun Y, Noordhuis P, Whiteman KR, et al. IMGN779, a Novel CD33-Targeting Antibody-Drug Conjugate with DNA- 
Alkylating Activity, Exhibits Potent Antitumor Activity in Models of AML. Mol Cancer Ther. 2018;17(6):1271-1279.

25. Cortes JE, Traer E, Wang ES, et al. IMGN779, a Next-Generation CD33Targeting Antibody-Drug Conjugate (ADC) Demonstrates Initial Antileukemia Activity in Patients with Relapsed or Refractory Acute Myeloid Leukemia. Blood. 2017;130(Suppl 1):1312-1312.

26. Tarantelli C, Gaudio E, Arribas AJ, et al. POR309 Is a Novel Dual PI3K/mTOR Inhibitor with Preclinical Antitumor Activity in Lymphomas as a Single Agent and in Combination Therapy. Clin Cancer Res. 2018;24(1):120-129.

27. Hicks SW, Lai KC, Gavrilescu LC, et al. The Antitumor Activity of IMGN529, a CD37Targeting Antibody-Drug Conjugate, Is Potentiated by Rituximab in Non-Hodgkin Lymphoma Models. Neoplasia. 2017;19(9):661-671.

28. Al-Katib AM, Aboukameel A, Mohammad R, Bissery MC, Zuany-Amorim C. Superior antitumor activity of SAR3419 to rituximab in xenograft models for non-Hodgkin's lymphoma. Clin Cancer Res. 2009;15(12):4038-4045.
29. Kantarjian HM, Lioure B, Kim SK, et al. A Phase II Study of Coltuximab Ravtansine (SAR3419) Monotherapy in Patients With Relapsed or Refractory Acute Lymphoblastic Leukemia. Clin Lymphoma Myeloma Leuk. 2016;16(3):139-145.

30. Yu SF, Zheng B, Go M, et al. A Novel AntiCD22 Anthracycline-Based Antibody-Drug Conjugate (ADC) That Overcomes Resistance to Auristatin-Based ADCs. Clin Cancer Res. 2015;21(14):3298-3306.

31. Deckert J, Park PU, Chicklas S, et al. A novel anti-CD37 antibody-drug conjugate with multiple anti-tumor mechanisms for the treatment of B-cell malignancies. Blood. 2013;122(20):3500-3510.

32. Przepiorka D, Ko CW, Deisseroth A, et al. FDA Approval: Blinatumomab. Clin Cancer Res. 2015;21(18):4035-4039.

33. Pulte ED, Vallejo J, Przepiorka D, et al. FDA Supplemental Approval: Blinatumomab for Treatment of Relapsed and Refractory Precursor B-Cell Acute Lymphoblastic Leukemia. Oncologist. 2018;23(11):13661371.

34. Jen EY, Xu $Q$, Schetter $A$, et al. FDA Approval: Blinatumomab for Patients with B-cell Precursor Acute Lymphoblastic
Leukemia in Morphologic Remission with Minimal Residual Disease. Clin Cancer Res. 2019;25(2):173-477.

35. O'Leary MC, Lu X, Huang Y, et al. FDA Approval summary: tisagenlecleucel for treatment of patients with relapsed or refractory B-cell precursor acute lymphoblastic leukemia. Clin Cancer Res. 2018 Oct 11. [Epub ahead of print]

36. Bouchkouj N, Kasamon YL, de Claro RA, et al. FDA Approval summary: axicabtagene ciloleucel for relapsed or refractory large Bcell lymphoma. Clin Cancer Res. 2018 Nov 9. [Epub ahead of print]

37. Chow VA, Shadman M, Gopal AK Translating anti-CD19 CAR T-cell therapy into clinical practice for relapsed/refractory diffuse large B-cell lymphoma. Blood. 2018;132(8):777-781

38. Jurczak W, Zinzani PL, Gaidano G, et al Phase IIa study of the CD19 antibody MOR208 in patients with relapsed or refractory B-cell non-Hodgkin's lymphoma. Ann Oncol. 2018;29(5):1266-1272.

39. Munshi PN, Ujiani C. The acceleration of CAR-T therapy in non-Hodgkin lymphoma. Hematol Oncol. 2018 Nov 14 [Epub ahead of print] 